Document downloaded from:

http://hdl.handle.net/10251/115607

This paper must be cited as:

Rubio Michavila, C.; Candelas Valiente, P.; Minin, IV.; Minin, OV.; Tarrazó-Serrano, D. (2019). Manipulation of focal patterns in acoustic Soret type zone plate lens by using reference radius/phase effect. Ultrasonics. 91:237-241. doi:10.1016/j.ultras.2018.07.022

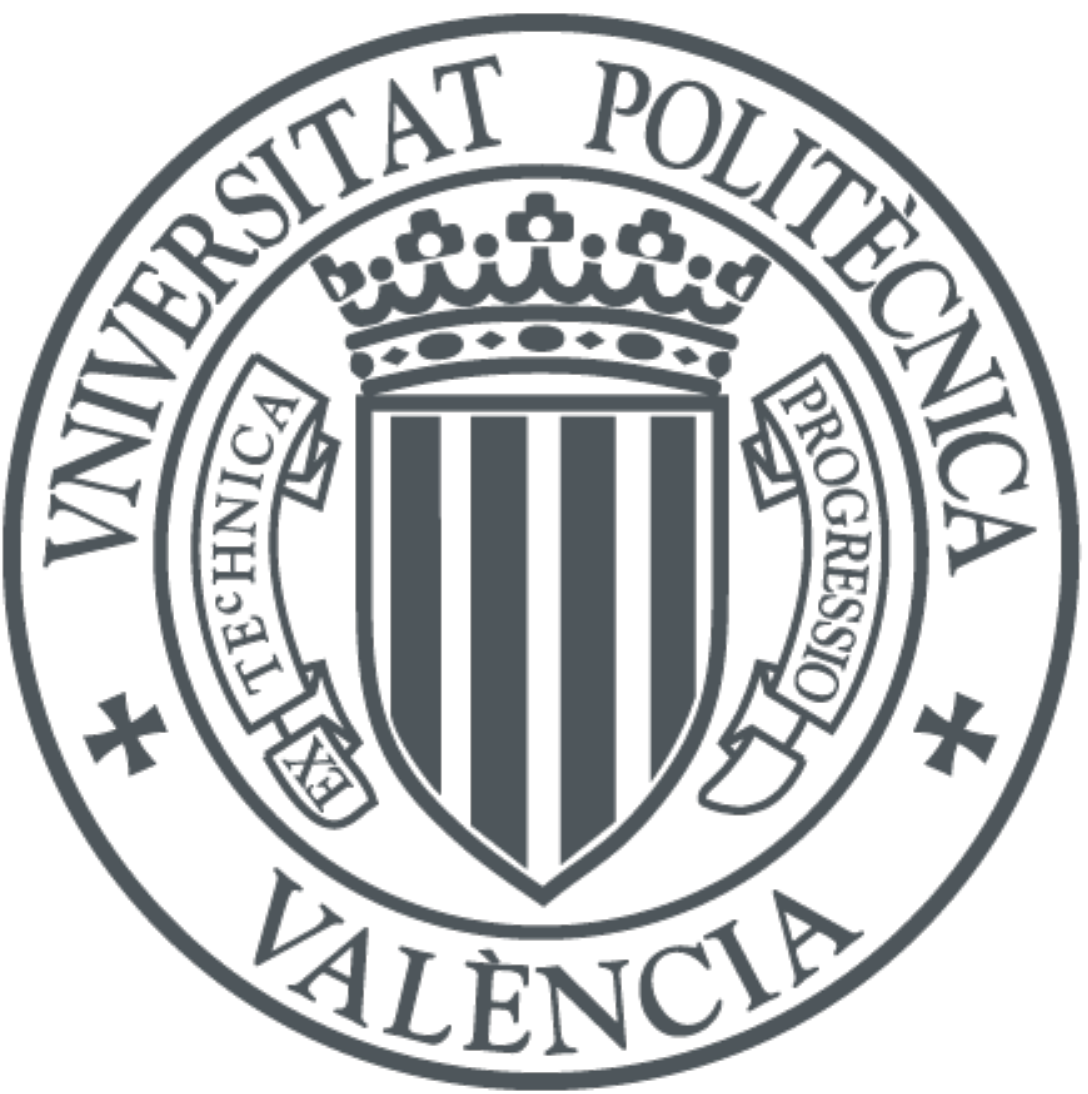

The final publication is available at

https://doi.org/10.1016/j.ultras.2018.07.022

Copyright Elsevier

Additional Information 


\title{
Manipulation of focal patterns in acoustic Soret type zone plate lens by using reference radius/phase effect
}

\author{
Daniel Tarrazó-Serrano ${ }^{1}$, Constanza Rubio ${ }^{1, *}$, Oleg V. Minin ${ }^{2}$, Pilar Candelas ${ }^{1}$, Igor V. Minin ${ }^{3}$ \\ ${ }^{1}$ Centro de Tecnologías Físicas: Acústica, Materiales y Astrofísica, Universitat Politècnica de València, Camino de Vera s/n, 46022 Valencia, Spain, UE \\ ${ }^{2}$ Tomsk State University, 30 Lenin Avenue, Tomsk, 634050, Russia \\ ${ }^{3}$ Tomsk State Politechnical University, 36 Lenin Avenue, Tomsk, 634050, Russia \\ * corresponding author: crubiom@fis.upv.es
}

\begin{abstract}
The manipulation of focal patterns of acoustic underwater Soret Zone Plate lens in far fields, such as manipulation (optimization) of Sidelobe Level and the design of long depth of focus by selecting the simple free parameter called reference radius (phase) has been demonstrated.Two effects have been studied by means of numerical simulations. Regarding the first effect, simulations demonstrate diffraction limited focal spot ( 0.47 wavelength) and $3 \mathrm{~dB}$ reduction of the first Sidelobe Level using Soret ZP with an optimal reference radius and without causing neither main lobe broadening or gain reduction. In the second effect, by using numerical simulations an increasing of depth of focus, more than 2 times, in comparison with classical Soret ZP with high numerical aperture $(\mathrm{F} / \mathrm{D}=2.5)$, was observed.
\end{abstract}

Keywords: Ultrasonic lenses, Soret Zone Plate, Side Lobel Level, Reference Radius PACS: 43.20.f, 43.35.Bf, 43.58.Ls,

\section{Introduction}

A lens is a device that can manipulate waves by taking advantage of both their refractive and diffractive properties to produce wave focalization. Acoustic lenses have attracted the attention

5 of scientists around the world for their application in multiple 35 areas of industry, engineering, acoustic tweezers, biomedicine and for other applications requiring low-cost acoustic focusing lenses [1-11], to mention a few. This explains why their design is subject of much research nowadays. One of the effects 10 of these lenses is their capability of focalization, which it can 40 be obtained by means of different types of methods and techniques.

Acoustic lenses based on phononic crystals have been designed and fabricated [12]. Other types of acoustic lenses are

15 those designed with a refractive index that varies throughout 45 the medium continuously. These are the so-called GradientIndex (GRIN) lens, and the variation of the refractive index is achieved by forming labyrinthine structures [13-15]. Acoustic metamaterials [16] and acoustic resonators [17] have also been used in the design of acoustic lenses.

There are also acoustic devices that focus sound through the phenomenon of diffraction. Acoustic lenses based on constructive interferences of diffracted fields, such as Fractal Lenses or Fresnel Zone Plates (FZP), are a good alternative to refractive lenses. FZP lenses have advantages in situations where ${ }_{55}$ size, weight, system complexity and fabrication are important $[18,19]$. The classic FZPs that are implemented alternating transparent to opaque Fresnel zones are called Soret Zone Plates (SZP) [20]. This work is focused on this type of Fresnel's lenses.

In Ref. [21] the authors study a lens formed by slits of different thickness located at $r_{n}$, while in the present work rigid scattered located at $r_{n}$ ( $r_{n}$ is a radius of n-th Fresnel zone) has been used. Following Babinet's principle considerations, both approaches can be considered equivalent. Recently Calvo et al. [22] fabricated and characterized an underwater acoustic SZP lens with alternating transparent and opaque zones made of soft silicone rubber. Calculations shown some reduction of the first Side Lobe Level (SLL), but with a main lobe broadening.

Although the diffraction of the acoustic field produce a maximum pressure field in the axis, the focal point shows some missdefinition due to the existence of secondary lobes [23-25]. In such a way, the relationship between the main and secondary lobe, known as SLL, is one of the main problems that SZPs can exhibit [26].

Even though the maximum energy is obtained at the Focal Length $\left(F_{L}\right)$, the SLL are not high enough with SZP lenses for applications where high precision is required. In this regard, a new technique used in biomedicine such as High Intensity Focused Ultrasound [27, 28], both in acoustical printer [8] or in acoustical particle manipulations [10] requires very precise targeting area so it is desirable to be able to control the lobes of the transmitted signal.

In other fields of physics, such as electromagnetism, this problem has already been raised. Minin et al [29] studied the possibility of altering the energy distribution function in a predetermined focus area. Recently, the authors themselves have shown that by choosing a radius of the first zone or a reference phase, non-standard values of the SZP radii can be obtained 
60 which improve the performance of the antennas and add a new application to the antennas zone plates [30] add and in commu-110 nications to preferentially suppress the clutter return relative to the desired main beam signal [31].

The present study investigates numerically an alternative SZP design to reduce or manipulate the lobes of the transmitted acoustic signal for an underwater SZP. A $\lambda / 6$, where $\lambda$ is the ${ }_{115}$ wavelength, thin SZP and 15 Fresnel zones width is thereby obtained in simulation showing different phenomena such as side lobe corrections and ultra-wide beam pattern. The simulations

70 have been carried out using the Finite Element Method (FEM) $[32,33]$.

\section{Theoretical Analysis and Simulation}

A SZP is structured in concentric circular sections known as Fresnel Regions. Each Fresnel Region has the same surface and 75 makes the same contribution to the focusing procedure. Consecutive Fresnel Regions have a $180^{\circ}$ phase shift between them and they interfere destructively. Among other, the main SZP design parameters to deal with are $F_{L}$ that is the location, in the axial coordinate, where the acoustic pressure field is focused; the Number of Fresnel Zones $(N)$ which include both opaque and transparent circular sections; the Design Frequency $(f)$, it is the frequency at which the lens is expected to focus at the $F_{L}$ (see Fig 1a). Once the design values have been set, the radial distances of the implemented lens can be obtained using the Eq.(1) which depends on all the previous design parameters and is valid for plane wave incidence.

$$
r_{n}=\sqrt{n \lambda F_{L}+\left(\frac{n \lambda}{2}\right)}
$$

On the other hand, in the earliest work was recognized the existence of a free parameter in the design of zone plates, where the radius of the central Fresnel zone were treated. This radius is known as the reference radius [29] and it is the new free pa- ${ }_{120}$ rameter. It should be note that the choice of the first zone as the reference is arbitrary. From the point of view of geometrical optics, the introduction of a reference radius does not change the properties of the zone plate. However, in the wave approximation this is not so. This reference radius is equal to introducing ${ }_{125}$ a variable reference phase [30].

In other words, another parameter that can be varied in calculating the modulation function of a zone plate is a permanent phase shift. The quasi-Fresnel radii can be displaced by adding an initial phase shift to the phase function of the SZP. ${ }_{130}$ This means in fact that the zone is divided not by starting at the extremum of the transmission phase function but at an arbitrary value of transmission [30]. In this case for binary zone plate the quasi-Fresnel radii will be defined by the Eq.(2) [30]:

$$
r_{n}=\sqrt{n \lambda \sqrt{F_{L}^{2}+r_{0}^{2}}+\left(\frac{n \lambda}{2}\right)^{2}+r_{0}^{2}}
$$

In order to study the physical phenomena involved in the interaction between the ultrasound waves and the lenses, it ${ }_{140}$ has been necessary to implement a mathematical model that replicates the characteristics of the system. Commercial FEM software COMSOL Multyphysics [34] has been used to determine the distribution of acoustic pressure and how the generated sources remain once the waves interact with the lens and diffract, causing the interference phenomena. FEM provides an approximate numerical solution of the model. This method performs a discretization of the model and solves the Helmholtz Partial Differential Equation (PDE) described in the equation Eq.(3).

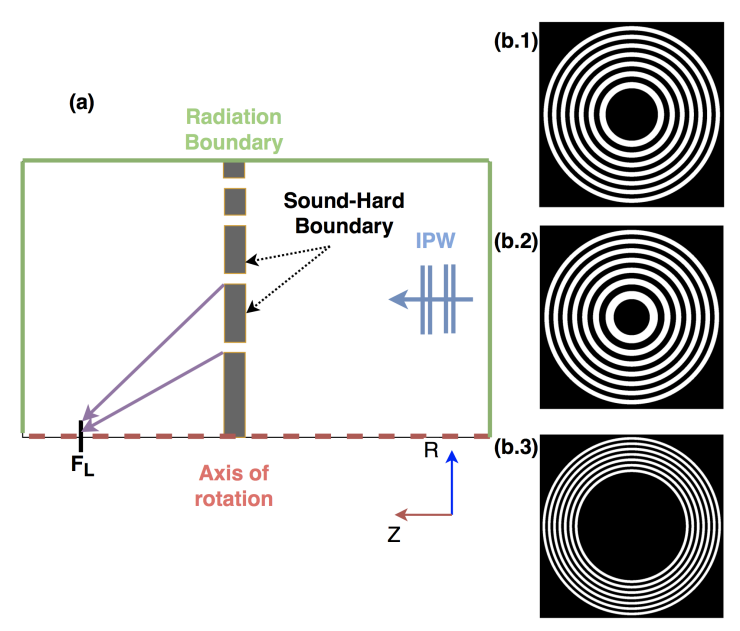

Figure 1: (a) FEM Scheme. In blue, the boundary of the Incident Pressure Wave (IPW), in green radiation condition boundaries and in red axis of rotation. SZP zones are considered rigid. (b.1) SZP with $r_{0}=0$ (b.2) Amplitude mask of SZP with $r_{0} \ll r_{1}$, (b.3) Amplitude mask of SZP with $r_{0} \gg r_{1}$. Note that for visual information the picture (b.2) is not in scale.

$$
\nabla\left(-\frac{1}{\rho} \nabla p\right)=\frac{\omega^{2}}{\rho c^{2}} p
$$

All the SZP lenses (including or not radius/phase reference) are axisymmetric, the model has been simplified by implementing a half-lens and applying a rotation with its central axis as the center for its complete generation. This facilitates the reduction of the degrees of freedom necessary to obtain the results of the numerical simulation and thus reducing the calculation time and its complexity. In this work, underwater acoustic transmission is considered. To solve the Helmholtz equation Eq.(3), typical values of water such as density of the medium $\left(\rho=1000 \mathrm{~kg} / \mathrm{m}^{3}\right)$ and sound propagation velocity $(c=1500 \mathrm{~m} / \mathrm{s})$ have been taken. The working frequency $(f)$ of both lenses is $250 \mathrm{kHz}$ (therefore the wavelength $(\lambda=6 \mathrm{~mm})$ and it can be found at Eq.(3) by its relationship with the angular velocity $(\omega)$. Finally, $p$ corresponds to the acoustic pressure. The initial conditions of the model were the following: Initial pressure $\left(p_{0}\right)$ was zero and the thickness of the lenses was 1 $\mathrm{mm}$ which was despicable with respect to the wavelength of the incident pressure wave. The defined boundary conditions were: the lenses were considered infinitely rigid marking the contours of the elements applying the Newmann condition (the sound velocity in the contour was zero); the contours of the 
model were defined as wave radiation condition boundary to emulate an infinitely large medium and therefore the Sommerfeld condition was satisfied; the axis of rotation was defined as axis of axisymmetry to simulate the $360^{\circ}$ rotation that makes up the SZPs in 3D. The geometry of the mesh used was triangular. A maximum element size of $\lambda / 8$ and a minimum of $\lambda / 14$ was established to avoid numerical dispersion in the simulations. In Fig. 1 the scheme of the model implemented in FEM is shown.

\section{Results}

The Numerical Aperture (NA) of the lens design must be taken into account. NA is defined as $F_{L}$ divided by the diameter of the lens $(D)$. It is well known that the Rayleigh resolution is determined by the numerical aperture of the lens and the wavelength of the radiation used. The smaller the NA, the better is the resolution of the lens. Taking into account that it must be designed with a small numerical aperture, a value NA of 0.214 has been selected for simulation. By selecting a focus position at $27 \mathrm{~mm}$ in axis of symmetry, a maximum lens diameter of $126_{180}$ $\mathrm{mm}$ is obtained that complies with the chosen NA.

Therefore, the design of the SZP will comply with these characteristics implemented in the construction equation Eq.(1), taking into account that $\lambda$ is $6 \mathrm{~mm}$ for the case of the host medium is water. To design the SZP with radius/phase refer- ${ }_{-185}$ ence, two cases are considered. Given the construction equation Eq.(2), a reference radius must be chosen for each case. The first one, is that $r_{0}$ is smaller than the first radius $\left(r_{1}\right)$ of the original SZP $\left(r_{0} \ll r_{1}\right)$. The second case occurs when $r_{0}$ is bigger than $r_{1}\left(r_{0} \gg r_{1}\right)$.

The results are expressed as a function of the Intensity Level (IL), that is defined as the ratio, in $\mathrm{dB}$, between the downstream intensity $I$ and the incident intensity $I_{\text {inc }}$.

$$
\text { Intensity Level }(d B)=10 \log \left(\frac{I}{I_{\text {inc }}}\right)
$$

\subsection{SLL Reduction}

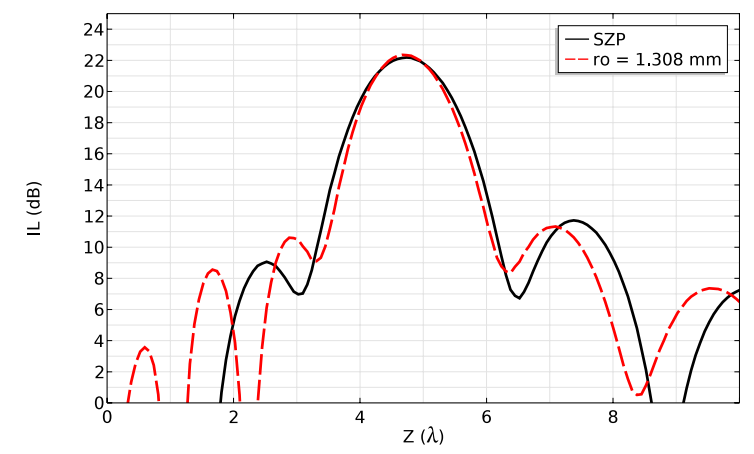

Figure 2: Numerical results of the Intensity Level (in $\mathrm{dB}$ ) along $\mathrm{Z}$ axis. ${ }^{195}$ Comparison between classical SZP and SZP with reference radius $r_{0}\left(r_{0}=\right.$ $1.308 \mathrm{~mm}$ ) being $r_{0} \ll r_{1}$. The results are shown for $f=250 \mathrm{kHz}$

In the first case, a reference radius smaller than the first raare shown below. In Fig. 2 a longitudinal section is shown for

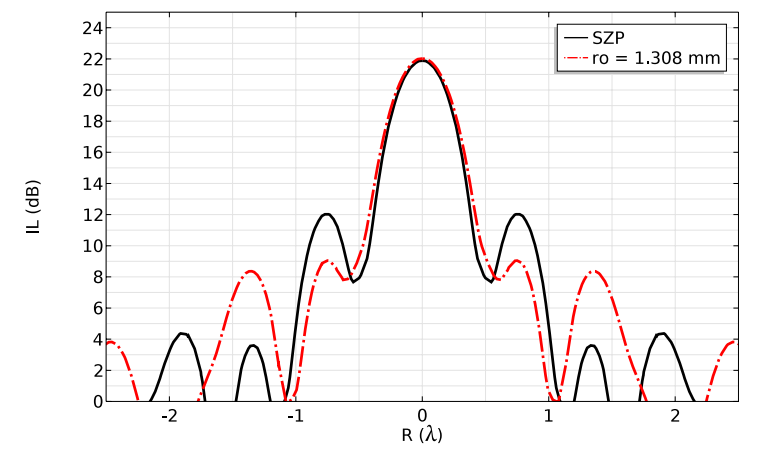

Figure 3: Numerical results of the Intensity Level (in $\mathrm{dB}$ ) along $\mathrm{R}$ axis. Comparison between classical SZP and SZP with reference radius $r_{0}\left(r_{0}=\right.$ $1.308 \mathrm{~mm}$ ) beeing $r_{0} \ll r_{1}$. The results are shown at the Focal Length $\left(F_{L}\right)$ for $f=250 \mathrm{kHz}$

the optimal case compared with the original SZP. It can be seen the position of the $F_{L}$ has not been modified regardless of the $r_{0}$ chosen. In Fig. 3 a cross section at the $F_{L}$ position is made, in other words a cut in $\mathrm{R}$ axis is made. In this case, it can be seen that there is a modulation of the secondary lobes. An SLL increase of approximately $3 \mathrm{~dB}$ is produced. By the conservation energy law, a displacement of this energy is caused towards other lobes. Further, the energy in $F_{L}$ is conserved. It is also followed from Fig.3 that FWHM for both cases is almost the same and equal to $0.47 \lambda$, which is near the diffraction limit.

In Table 1, a comparison is shown for different cases that meet the condition $r_{0} \ll r_{1}$. As $r_{0}$ decreases, SLL increases until a limit value is obtained. Therefore, $r_{0}=r_{1} / 10$ has been selected as the optimum value since the SLL cannot be improved for selected values of NA.

Table 1: Numerical values of Side Lobe Level (SLL) and Full Width Half Maximum (FWHM) for different values of the reference radius. Note that $r_{0}=0$ corresponds to SZP and the FWHM is obtained along R axis.

\begin{tabular}{|c|c|c|}
\hline$r_{0}(\mathrm{~mm})$ & SLL $(\mathrm{dB})$ & FWHM $(\lambda)$ \\
\hline \hline 0 & 9.9 & $0.46 \pm 0.12$ \\
\hline $6.540\left(r_{1} / 2\right)$ & 11.8 & $0.47 \pm 0.12$ \\
\hline $4.359\left(r_{1} / 3\right)$ & 12.4 & $0.47 \pm 0.12$ \\
\hline $2.615\left(r_{1} / 5\right)$ & 12.8 & $0.48 \pm 0.12$ \\
\hline $1.308\left(r_{1} / 10\right)$ & 13.0 & $0.48 \pm 0.12$ \\
\hline $0.654\left(r_{1} / 20\right)$ & 13.0 & $0.48 \pm 0.12$ \\
\hline $0.436\left(r_{1} / 30\right)$ & 13.0 & $0.48 \pm 0.12$ \\
\hline
\end{tabular}

\subsection{Focus Expansion}

In the second case, values are taken above the first radius of the classical SZP. A longitudinal cut has been made in the same way as in the previous case that corresponds to the $\mathrm{Z}$ axis. It can be seen that despite maintaining the position of the maximum energy in the $F_{L}$, as $r_{0}$ increases, an energy expansion in the longitudinal axis is caused. In Fig.4, several higher reference radii than $r_{1}$ can be observed.

It could be noted that by selecting the reference radius, it may be controlled not only the first sidelobes (Fig.3) but also 


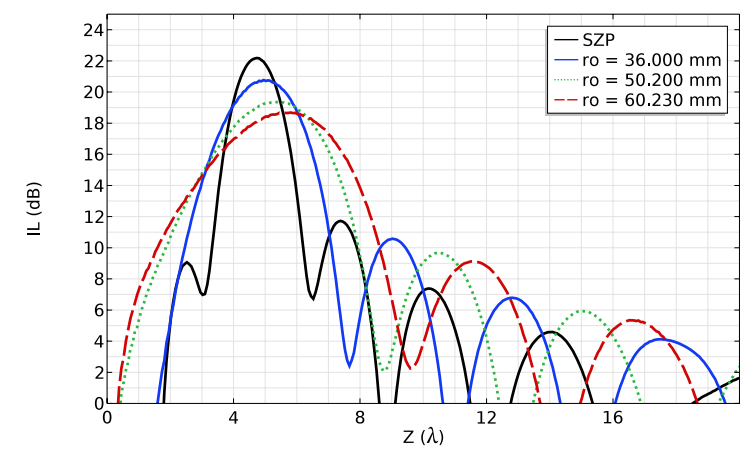

Figure 4: Numerical results of the Intensity Level (in $\mathrm{dB}$ ) along $\mathrm{Z}$ axis. Comparison between classical SZP and SZP with reference radius $r_{0}$ that accomplish $r_{0} \gg r_{1}$ for $f=250 \mathrm{kHz}$

the main beamwidth to form ultra wide main beam. In Table 2, the value of the FLHM along the $\mathrm{Z}$ axis and the focal shift have been shown.The focal shift in SZP is completely dependent on the Fresnel number $\left(N_{F}\right)$, which is determined by [35]

$$
N_{F}=\frac{D^{2}}{4 \lambda F_{L}}
$$

As it can be seen from Fig.1 and formula Eq.(5), increasing the effective lens size (D), or reducing the focal length $\left(F_{L}\right)$ or decreasing the working wavelength will increase the Fresnel ${ }^{230}$ number Eq.(5), and thus, reduce the focal shift. Also in our design of SZP the focal shift increases with reference radius increasing. This effect was not previously observed.

For a reference radius of $r_{0}=50.20 \mathrm{~mm}$, the intensity referred to the incident intensity $\left(I / I_{\text {inc }}\right)$ at the focus and at two points of ${ }^{235}$ half intensity drop (FLHM) along $\mathrm{Z}$ axis is shown in Fig.5. The inset of the figure shows an $I / I_{\text {inc }}$ map. White discontinuous line point out the R axis cut which corresponds to FLHM points.

Table 2: Numerical values of Full Length Half Maximum (FLHM), Focus Shift and maximum Intensity referred to incident Intensity $\left(I_{\max } / I_{i n c}\right)$ for diferent values of the reference radius along $\mathrm{Z}$ axis . Numerical value obtained for SZP designed $F_{L}$ corresponds to $4.65 \lambda$.

\begin{tabular}{|c|c|c|c|}
\hline$r_{0}(\mathrm{~mm})$ & FLHM $(\lambda)$ & Focus Shift $(\lambda)$ & $I_{\max } / I_{\text {inc }}$ \\
\hline \hline 0 & 1.53 & 0 & 170 \\
\hline $36.00\left(r_{6}\right)$ & 2.56 & +0.30 & 118 \\
\hline $50.20\left(r_{10}\right)$ & 3.48 & +0.55 & 86 \\
\hline $60.23\left(r_{13}\right)$ & 4.16 & +1.05 & 73 \\
\hline
\end{tabular}

\section{Discussion and Conclusions}

A numerical study of the characteristics of the acoustic SZP with radius/phase reference has been carried out. Two cases have been studied in which two different phenomena are caused. As expected, a SLL reduction has been achieved when255 $r_{0}$ is minor than $r_{1}$. This is due to the control of constructive interference of diffracted waves from circular zones' array. By

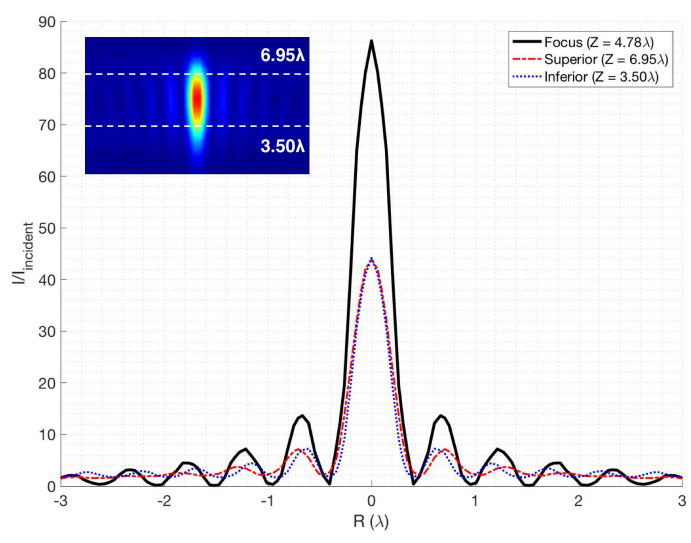

Figure 5: Intensity the intensity referred to the incident intensity $\left(I / I_{i n c}\right)$ at the focus $(Z=4.78 \lambda)$ and two points of half intensity (FLHM). Red dashed line corresponds to $Z=6.95 \lambda$ and blue dotted line to $Z=3.50 \lambda$. The inset shows an $I / I_{\text {inc }}$ map and the cut lines of FLHM for a SZP with a reference radius of $r_{0}=$ $50.20 \mathrm{~mm}$.

changing the reference radius, the spatial frequency of the Fresnel zones has been changed. Having control over spatial frequency means being able to obtain a much finer focus, which in the case of applications such as HIFU, is of great interest as it allows to obtain a more precise foci.

In the case of being $r_{0}$ bigger than $r_{1}$, an expansion of the focus along the longitudinal axis is caused. This is due to 2 effects: one is the same as in the first case and the second one is amplitude apodisation effect. The latest is due to geometrical effect interpretation of central apodisation (obturation) on background sound, as the focus at the first diffraction order is in the shadow of the obturation for all other diffraction orders. Acoustic wave from negative orders is divergent and acoustic wave from positive orders above one, is rejected far from the center of the SZP [36]. That is, a small fraction of the first order radiation is shifted to the higher order lobes.

To the best of our knowledge, the two designed and characterized focusing properties in this work, regarding the reference radius had been never studied previously in acoustics SZP lenses, making this work a great contribution to the field. Having these tunable properties will allow Fresnel lenses to be improved and further research to be made, given their possible applications in situations where better precision is required. For instance, the prospect of these results are important in some medical applications, where depending on the tumor or target area it is desirable either to have a finer focus, or an expansion of the focus.

\section{Acknowledgements}

The research is carried out at Tomsk Polytechnic University within the framework of Tomsk Polytechnic University Competitiveness Enhancement Program.

This work has been supported by TEC2015-70939-R (MINECO/FEDER). 


\section{References}

[1] Kamaljit Vilkhu, Raymond Mawson, Lloyd Simons, and Darren Bates. Applications and opportunities for ultrasound assisted extraction in the food industrya review. Innovative Food Science $\mathcal{E}$ Emerging Technologies, 9(2):161-169, 2008

[2] S Albu, E Joyce, L Paniwnyk, JP Lorimer, and TJ Mason. Potential for the use of ultrasound in the extraction of antioxidants from rosmarinus officinalis for the food and pharmaceutical industry. Ultrasonics Sonochemistry, 11(3-4):261-265, 2004.

[3] Ji-Tai Li, Jun-Fen Han, Jin-Hui Yang, and Tong-Shuang Li. An efficient synthesis of 3, 4-dihydropyrimidin-2-ones catalyzed by nh2so3h under ultrasound irradiation. Ultrasonics Sonochemistry, 10(3):119-122, 2003.

[4] P. Pignoli, E. Tremoli, A. Poli, P. Oreste, and R. Paoletti. Intimal plus medial thickness of the arterial wall: A direct measurement with ultrasound imaging. Circulation, 74(6):1399-1406, 1986.

[5] RO Illing, JE Kennedy, F Wu, GR Ter Haar, AS Protheroe, PJ Friend, FV Gleeson, DW Cranston, RR Phillips, and MR Middleton. The safety and feasibility of extracorporeal high-intensity focused ultrasound (hifu) for the treatment of liver and kidney tumours in a western population. British journal of cancer, 93(8):890, 2005.

[6] D.M. McCann and M.C. Forde. Review of ndt methods in the assessment ${ }_{350}$ of concrete and masonry structures. NDT and E International, 34(2), 2001.

[7] D. C. Calvo, A. L. Thangawng, M. Nicholas, and C. N. Layman. Thin fresnel zone plate lenses for underwater acoustics: Modeling and experiments. OCEANS'15 MTS/IEEE Washingtonl, (October), 2015.

[8] Isao Amemiya, Hitoshi Yagi, Kenichi Mori, Noriko Yamamoto, Shiro Saitoh, Chiaki Tanuma, and Shuzo Hirahara. Ink Jet Printing with Focused Ultrasonic Beams. Recent Progress in Ink Jet Technologies II, volume 5. Society for Imaging Science and Technology, 1999.

[9] SF Hon, KW Kwok, HL Li, and HY Ng. Self-focused acoustic ejectors for viscous liquids. Review of scientific instruments, 81(6):065102, 2010.

10] You-Lin Tu, Shih-Jui Chen, and Yean-Ren Hwang. Design of fresne lens-type multi-trapping acoustic tweezers. Sensors, 16(11):1973, 2016.

[11] Ethan Schonbrun, Charles Rinzler, and Kenneth B Crozier. Microfabricated water immersion zone plate optical tweezer. Applied Physics Let ${ }_{365}$ ters, 92(7):071112, 2008.

295 [12] F. Cervera, L. Sanchis, J. V. Sánchez-Pérez, R. Martínez-Sala, C. Rubio, F. Meseguer, C. López, D. Caballero, and J. Sánchez-Dehesa. Refractive Acoustic Devices for Airborne Sound. Physical Review Letters, 88(2):4, 2002.

13] Yong Li, Bin Liang, Xu Tao, Xue Feng Zhu, Xin Ye Zou, and Jian Chun Cheng. Acoustic focusing by coiling up space. Applied Physics Letters, 101(23), 2012.

[14] John T. Welter, Shamachary Sathish, Daniel E. Christensen, Philip G. Brodrick, Jason D. Heebl, and Matthew R. Cherry. Focusing of longitudinal ultrasonic waves in air with an aperiodic flat lens. The Journal of the Acoustical Society of America, 130(5):2789-2796, 2011.

[15] Pai Peng, Bingmu Xiao, and Ying Wu. Flat acoustic lens by acoustic grating with curled slits. Physics Letters, Section A: General, Atomic and Solid State Physics, 378(45):3389-3392, 2014.

[16] Sébastien Guenneau, Alexander Movchan, Gunnar Pétursson, an S. Anantha Ramakrishna. Acoustic metamaterials for sound focusing and confinement. New Journal of Physics, 9, 2007.

[17] Miguel Moleron, Marc Serra-Garcia, and Chiara Daraio. Acoustic Fresnel lenses with extraordinary transmission. Applied Physics Letters, 105(11), 2014.

[18] Donald C O'Shea, Thomas J Suleski, Alan D Kathman, and Dennis W Prather. Diffractive optics: design, fabrication, and test, volume 62. Spie Press Bellingham, WA, 2004.

[19] Shu Zhang, Leilei Yin, and Nicholas Fang. Focusing ultrasound with an acoustic metamaterial network. Physical Review Letters, 102(19), 2009.

[20] JL Soret. Ueber die durch kreisgitter erzeugten diffractionsphänomene. Annalen der Physik, 232(9):99-113, 1875.

[21] Gregory T. Clement, Hideyuki Nomura, and Tomoo Kamakura. Ultrasound Field Measurement Using a Binary Lens. IEEE Transactions on Ultrasonics, Ferroelectrics and Frequency Control, 62(2):350-359, 2015.

[22] David C. Calvo, Abel L. Thangawng, Michael Nicholas, and Christopher N. Layman. Thin Fresnel zone plate lenses for focusing underwater sound. Applied Physics Letters, 107(1), 2015.
23] A G Bashford, D W Schindel, D A Hutchins, and W M D Wright. Field characterization of an air-coupled micromachined ultrasonic capacitance transducer. Journal of the Acoustical Society of America, 101(1):315322, 1997.

[24] I. N. Kanevsky. Focusing of Sound and Ultrasound Waves [in Rusian]. Nauka, Moscow, 1977.

[25] Jun-Ichi Kushibiki ed by Hiroshi Shimizu, Noriyoshi Chubachi. Acoustical Imaging v.17. Plenum Press, New York and London, 1989.

[26] Igor V Minin and Oleg V Minin. Basic principles of fresnel antenna arrays, volume 19. Springer Science \& Business Media, 2008.

[27] Jean Francois Aubry, Kim Butts Pauly, Chrit Moonen, Gailter Haar, Mario Ries, Rares Salomir, Sham Sokka, Kevin Michael Sekins, Yerucham Shapira, Fangwei Ye, Heather Huff-Simonin, Matt Eames, Arik Hananel, Neal Kassell, Alessandro Napoli, Joo Ha Hwang, Feng Wu, Lian Zhang, Andreas Melzer, Young sun Kim, and Wladyslaw M. Gedroyc. The road to clinical use of high-intensity focused ultrasound for liver cancer: Technical and clinical consensus, 2013.

[28] Jean-Michel Escoffre and Ayache Bouakaz. Therapeutic ultrasound, volume 880. Springer, 2015.

[29] IV Minin and OV Minin. Control of focusing properties of diffraction elements. Soviet Journal of Quantum Electronics, 20(2):198, 1990.

[30] Igor V Minin and Oleg V Minin. Reference phase in diffractive lens antennas: A review. Journal of Infrared, Millimeter, and Terahertz Waves, 32(6):801, 2011.

[31] GW Webb, IV Minin, and Oleg V Minin. New technique to suppress sidelobe clutter in perimeter security systems. International Journal of High Speed Electronics and Systems, 17(02):367-382, 2007.

[32] SC Chan, C Mina, SS Udpa, W Lord, L Udpa, and T Xue. Finite element modeling of binary acoustic fresnel lenses. NDT and E International, 4(29):243, 1996.

[33] Olgierd Cecil Zienkiewicz, Robert Leroy Taylor, Olgierd Cecil Zienkiewicz, and Robert Lee Taylor. The finite element method, volume 3. McGraw-hill London, 1977.

[34] COMSOL Multiphysics. Comsol multiphysics user guide (version 4.3 a). COMSOL, AB, pages 39-40, 2012

[35] Yajun Li and Emil Wolf. Focal shifts in diffracted converging spherical waves. Optics Communications, 39(4):211-215, 1981.

[36] Robert J Vanderbei, David N Spergel, and N Jeremy Kasdin. Spiderweb masks for high-contrast imaging. The Astrophysical Journal, 590(1):593, 2003 